\title{
Improved image reconstruction in small animal PET using a priori estimates of single-pixel events
}

\author{
S. España, J. L. Herraiz, E. Vicente, E. Herranz, J. J. Vaquero, M. Desco, and J. M. Udias
}

\begin{abstract}
Most small animal PET scanners are based on arrays of pixelated scintillators crystals. As the read-out of individual pixels would be too expensive, identification of the crystal of interaction is usually made by center of energy methods based for instance on Anger logic. This allows for a reduction in the number of signals to be acquired, but prevents the identification of multi-hit events, that is, events in that one (or several) photon produces several hits in the detector, thus blurring the correct positioning of the interaction. Improving the identification of the pixel of interaction is pursued in this work by combining all the information acquired by the scanner without increasing the number of signals. The probability for every individual event for being single or multi-pixel is estimated from the $X Y$ positioning and energy information. This probability is fed into a 3D-OSEM iterative statistical reconstruction method. Every coincidence event detected may be analyzed combining information such as deposited energy, PMT XY location, time difference between both singles of the coincidence and coincidence and single rates, if available. With the proposed method, improved peak/noise ratio and better resolution are obtained without the introduction of additional hardware.
\end{abstract}

\section{INTRODUCTION}

$\mathrm{M}_{\mathrm{p}}$ OST small animal PET scanners are based on arrays of pixelated scintillators crystals. Read out of individual crystals would be too expensive and thus the identification of the crystal of interaction is usually made by center of energy methods based for instance on the Anger logic. This provides with $\mathrm{X}$ and $\mathrm{Y}$ signals for the positioning of the interaction of each event and look up tables can built that assign events to individual crystal pixels. Clearly, this method results in erroneous crystal identification (that deteriorates the image obtained) if the event actually corresponds to multiple hits in the detector due to, for instance, inter crystal scatter or detector photon pile-up.

Most often the method for assigning events to individual pixel elements is fully deterministic, in the sense that every accepted event is attributed to one and only one crystal with $100 \%$ certainty. The criteria for accepting events can be tightened to reduce the multi-hit contribution to the acquisitions, for instance using more restrictive lookup tables (LUT) that do not assign (accept) events that do not fall into

S.España is with the Grupo de Física Nuclear at Department of Atomic, Molecular and Nuclear Physics, Complutense University of Madrid, 28040 SPAIN (telephone: +34-913944484, e-mail: samuel@nuclear.fis.ucm.es).

J. L. Herraiz e-mail: joaquin@nuclear.fis.ucm.es

E.Vicente email: evicente@nuclear.fis.ucm.es.

E. Herrnaz e-mail: eherranz@nuclear.fis.ucm.es.

J.J.Vaquero email: juanjo@mce.hggm.es.

M. Desco e-mail: desco@mce.hggm.es.

J.M.Udias e-mail: jose@nuc2.fis.ucm.es. the (narrow) predetermined XY range for each crystal [1]. We propose here an alternative method that makes full use of the information obtained by the scanner for every coincidence event, namely XY position estimates and deposited energy in the detector, in order to improve the quality of the reconstructed images. Our method uses a kind of fuzzy logic, where every coincidence is not just accepted or rejected according to the fulfillment of certain energy and XY range restrictions in a binary logic way but rather, with the aid of extensive comparisons to real and simulated data, a combination of both energy and position information is built that represents the likely-hood for the event for being singlecrystal,. Indeed, the combined criteria is adjusted to yield the right estimates for single-hit events at different count rates, compared to realistic simulations [3]. Events identified as having a high likelihood of coming from a single-hit interaction in the detector are given above average reliability, while others are considered as being less reliable. The assignation of measured counts to individual crystals in this method is thus not just 0 or 1 but any number in between, depending to the likelihood of the count for being singlecrystal.

\section{METHOD}

Every coincidence event detected can be analyzed with the known information such as deposited energy, PMT XY location from Anger logic and coincidence and single rates, if available. In the most conventional procedure, the criteria to accept a coincidence as a valid one consist of fixing an energy window, a time coincidence window and a crystal map for the PMT location. In order to fully accept a coincidence it must fall inside all preset windows or otherwise it would be fully discarded. In this way, all counts that fit inside these chosen windows are considered equally good and the improvement in the image quality is obtained due to the fact that, on average, the quality of the counts (in terms of coming from scatter, being multi hit or suffering from pile-up effects) inside the window (energy, LUT or other) is better that the one of the counts outside it. Improved rejection of pile-up and intercrystal events can be achieved, as some groups have proposed, by a reduction of acceptable regions in the XY detector space, using only the regions of high intensity in the flood image of the detector [1]. Restricting to the region near the peaks helps to improve resolution due to the fact that the average quality or goodness of the counts that fulfill the more stringent criteria is larger, but at the cost of much reduced sensitivity. Furthermore, all regions receive contributions from both single-pixel and multi-pixel events and, even with more 
restrictive LUT and energy windows, single and multiple hits events are unavoidably employed in the reconstruction, with the same weight.

A more sophisticated elaboration of the information would consist of estimating the reliability or goodness for each single event and proceed through the list of events so that these estimates for the reliability of events are incorporated in the counts binning (for instance sinogram based) procedure. The estimate for an event for being single hit can be obtained from simulations tuned up to reproduce the features of the data acquired with the scanner and this probability can be employed, in the most simple version of this procedure, to bin effective counts where every event contributes a fraction of a count proportional to the above mentioned reliability.

It's needed to take into account that the ratio of single/multiple hits present in a particular acquisition depends strongly on the count rate, as pile up increases rapidly with activity and thus these estimates must depend on the acquisition rate (see Figs 1 and 2).
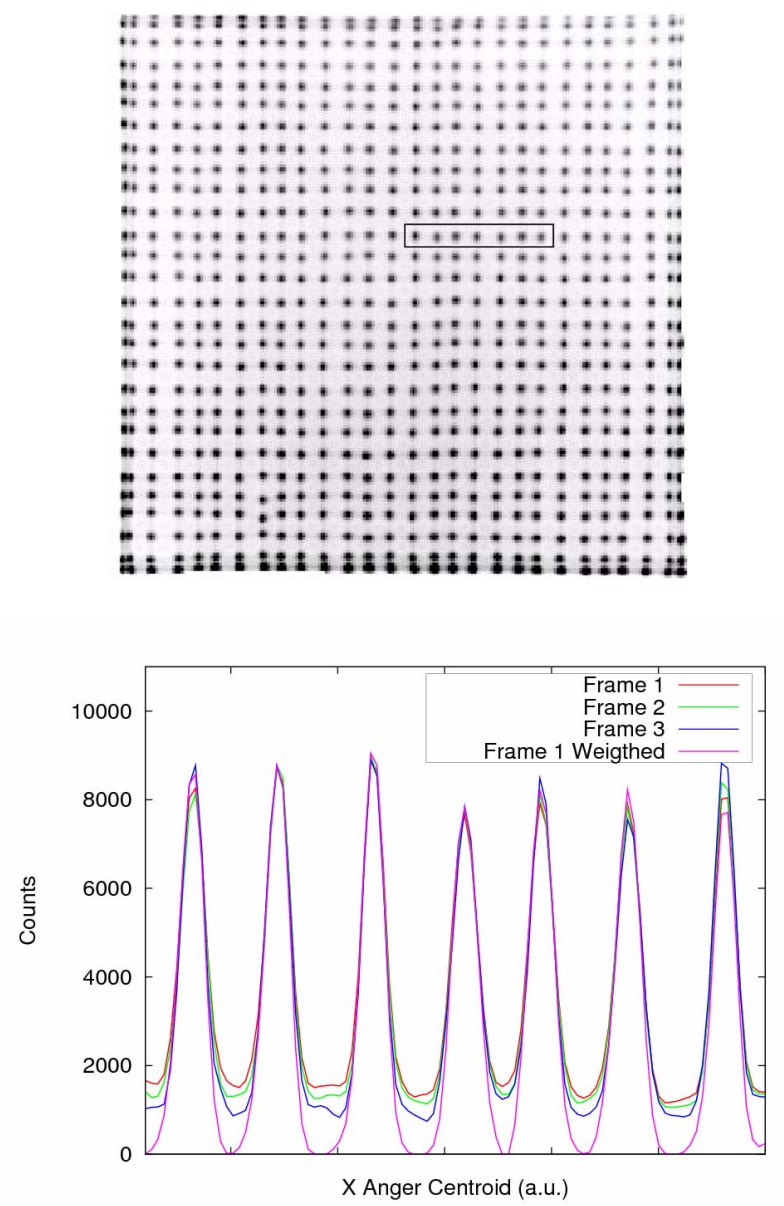

Fig. 1. Profile of flood image for one detector in a multiframe acquisition of a decaying FDG Micro Deluxe Resolution phantom acquired with the rPET scanner. Every frame are 30 minutes long and start two hours from the previous one. The profiles are normalized to the same peak values. An extra line (pink) shows the flood field profile after binning the counts with fractional values according to the probability of each event for being a singlehit one. Notice that the 'backgorund' baseline level due to photon detector pile-up and multi-hit events is removed by the weighting procedure.

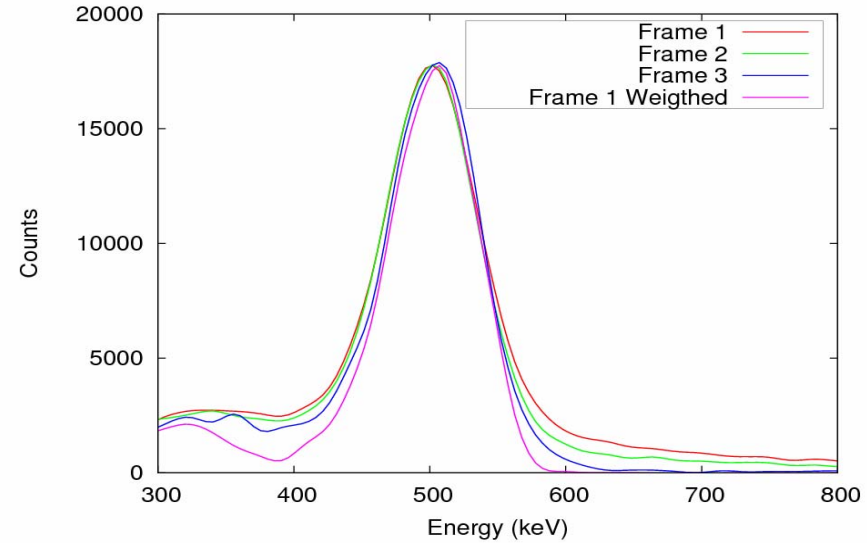

Fig. 2. Energy spectra for the same acquisition of Fig. 1, normalized to the same peak values for all the frames. Again, an extra line (pink) shows the energy spectrum for Frame 1 after weighting every count with its with singlehit likelyhood. We can see that this procedure effectively removes the high energy tail due to photon pile-up at the detector and background due to these events making the Compton edge visible.

To illustrate how the procedure works with real data, a multiframe acquisition of a decaying FDG phantom was performed with the rPET (SUINSA Medial Systems) [4] small animal high resolution scanner. Results for PMT XY positioning and energy spectrum are shown in Figs. 1 and 2. Frame 1 starts with an activity of $0.3 \mathrm{mCi}$ and has a duration of 30 minutes. Frame 2 starts two hours later and has the same duration. It can be seen the different pedestal level among peaks due to different pile-up at the two rates. Energy information can be combined with the $\mathrm{XY}$ position information, taken advantage that the probability of an event being single-hit depends strongly on the total energy in the detector: Inter-crystal scatter, single-crystal, pile-up and scatter before reaching the detector events display different energy spectrum at the detector. Coincidence and single-event rates dictate the levels of random and pile-up contribution with respect to the total number of events. Exhaustive analysis of real and simulated [3] data at different levels of activity and phantom sizes have been performed, obtaining an accurate characterization of system behavior. As an example of the information compiled from real acquisitions, the different baseline or pedestal levels for two real acquisitions with the rPET scanner are shown in Fig. 1. This information can be combined with results from simulations, such as those shown in Fig. 3, of the fraction of photons that interacts via singlepixel events in rPET, as a function of the energy deposited by the photon in the detector. 


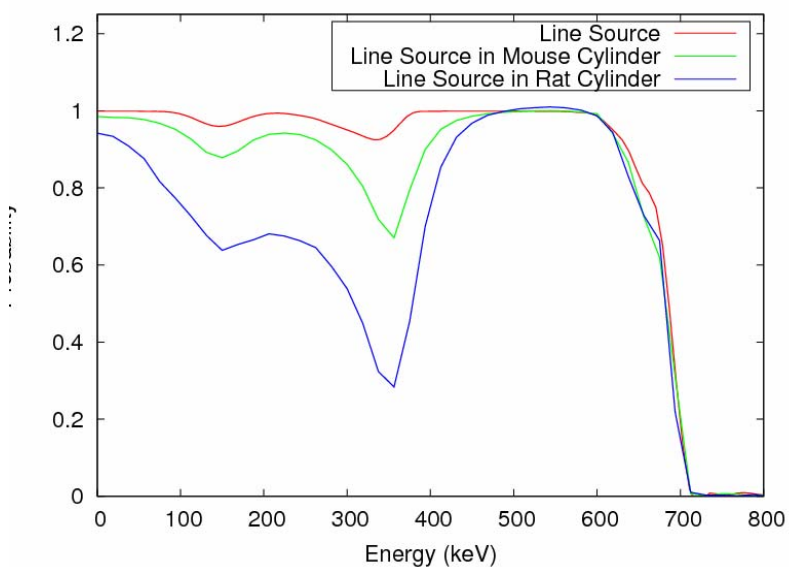

Fig. 3. Fraction of single-crystal photons versus total energy deposited. Results were obtained from simulations for the rPET scanner performed with PeneloPET [3] for three different phantoms.

As it has been already mentioned, to improve the quality of the images, the reliability of a particular event is computed from the a priori estimate of the probability for such event coming from a single-crystal interaction. This information is fed into an iterative reconstruction 3D-OSEM code [5]. It must be notice that while this guarantees that the image will benefit the most from the additional information, a simple version of this procedure, where effective counts are binned before reconstruction, would allow to use this method with non interactive reconstruction algorithms.
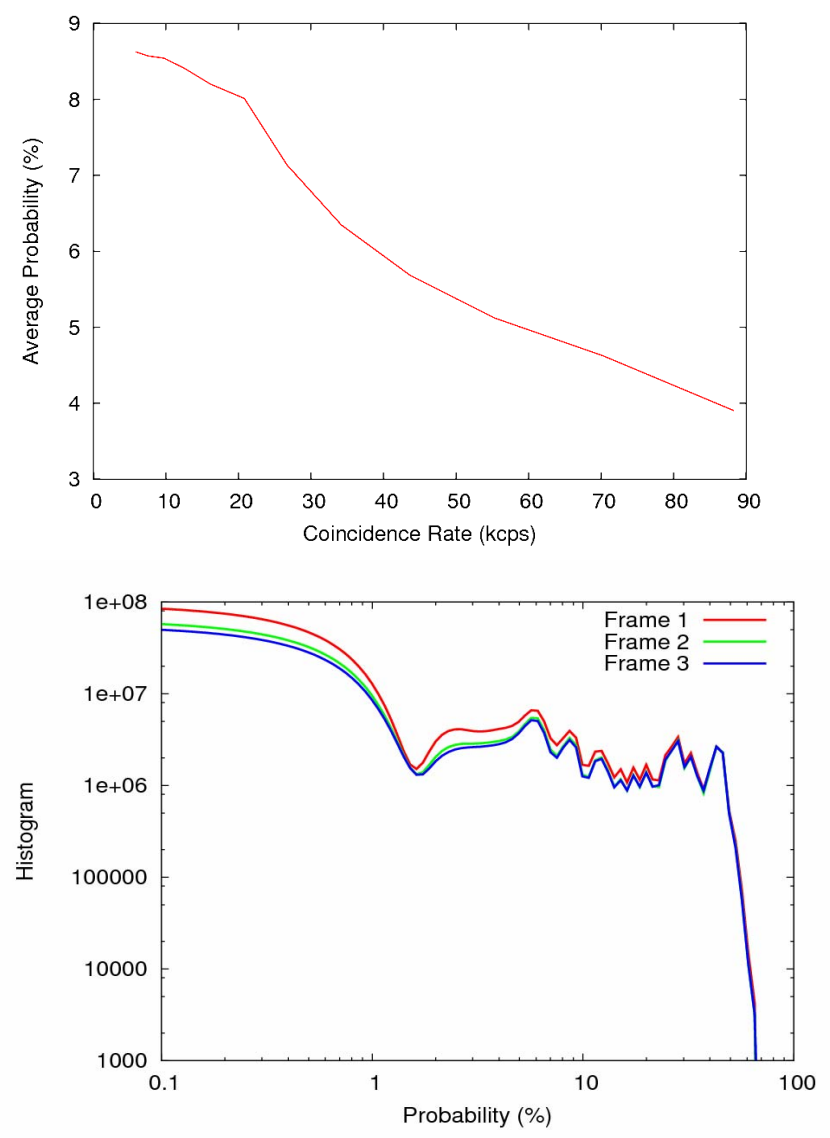

Fig. 4. (Top) Average values of the goodness probability of the coincidences for a multiframe acquisition versus the measured coincidence rate. High count rate acquisitions have a reduced average goodness due to pulse pile-up and random coincidences contribution. (Bottom). Histogram of goodness values for every count for the three frames at different activity levels of Figs. 1 and 2. Notice that no event has $100 \%$ probability of being singlecrystal.

The method is illustrated with acquisitions from the rPET scanner. Standard 3D-OSEM and improved 3D-OSEM reconstructions that employ the described procedure are compared. Resolution vs. noise and recovery coefficients (RC) were determined from a IQ phantom acquisition.

\section{RESULTS}

To asses the improvement in image quality achieved with the proposed method, we compare results of standard data processing where events are filtered and assigned using discrete LUT and energy tables so that they are accepted only if they fall inside an energy window of $400-600 \mathrm{keV}$. A Micro Deluxe Resolution phantom acquired for 45 minutes and with an initial activity of $1 \mathrm{mCi}$, with the $\mathrm{rPET}$ scanner and reconstructed with both 3D-OSEM methodologies is compared in Fig. 6. From the resulting images, it can be seen that an increased peak/background ratios and a improvement in spatial resolution are obtained with the new method.

In Fig. 4 it is shown the noise versus resolution figure of merit for both 3D-OSEM methods. Resolution is measured from the $2.5-\mathrm{mm}$ diameter capillary of the IQ phantom, taking derivatives of many profiles along these capillary and fitting the resulting profiles to gaussian functions. The FWHM for these gaussian, averaged over all possible profiles, is taken as a measure of resolution. For noise, several ROIS in the uniform region of the phantom are taken and the ratio of the standard deviation over the average value of all the voxels in these ROI is considered as a measure of noise. It can be noticed that a better resolution can be obtained with the proposed method beyond the one of the conventional one. On the other hand, the same image resolution can be obtained with fewer iterations with the improved method compared to the standard one, that is, convergence is accelerated and can be achieved with less computing effort.

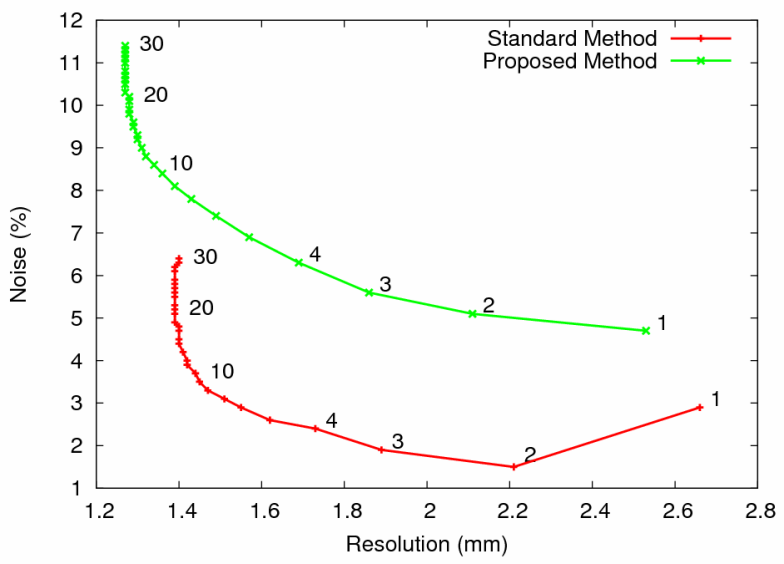




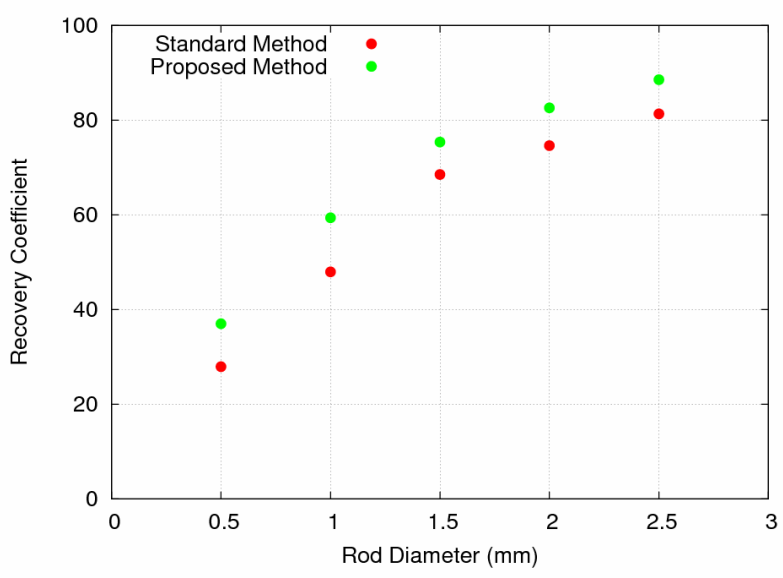

Fig. 5. Noise versus resolution plot (top) shows an increase of maximum achievable resolution for the improved method as well as a faster convergence. The labels over the lines indicate the number of iterations. Recovery coefficients versus rod diameter plot (bottom) shows also better results for the improved method.

That is, resolution recovery requires less iterations for the improved method, that also reaches a better resolution value than the standard one. Best resolution (FWHM) is $1.4 \mathrm{~mm}$ for the standard method and $1.25 \mathrm{~mm}$ for the improved one (Left). It must be also notice that there is a certain increase of the noise in the image with the improved method, due to the fact that a fraction of counts are effectively removed from the acquisition (see Fig. 4) due to their very low probability of being single-hit. In the uniform regions, where resolution (that is, good quality of the counts) is irrelevant, the removal of these counts increases the noise.

In Fig. 5 the recovery coefficients (RC) for the five capillary of the IQ phantom are compared for both reconstruction methods. There is an important increase in the $\mathrm{RC}$ values (by $10 \%$ of more). Visual inspection of reconstructed images of (left) mouse (heart region) and Micro Deluxe Resolution phantom also show better resolution and peak to noise ratios for the improved 3D-OSEM, confirming the findings of increased resolution and $\mathrm{RC}$.
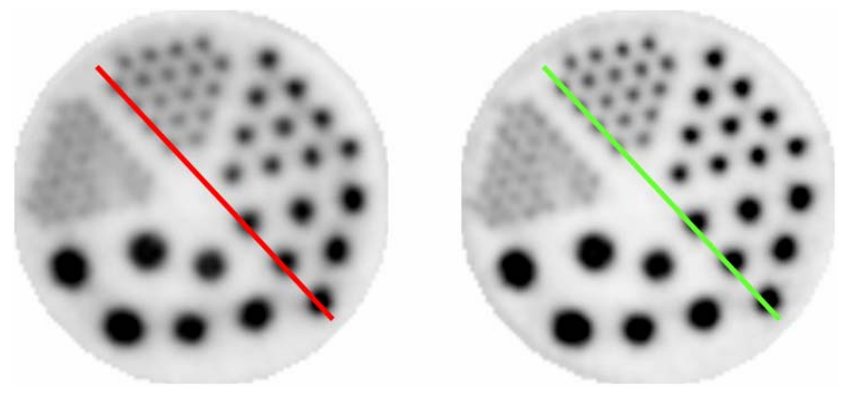

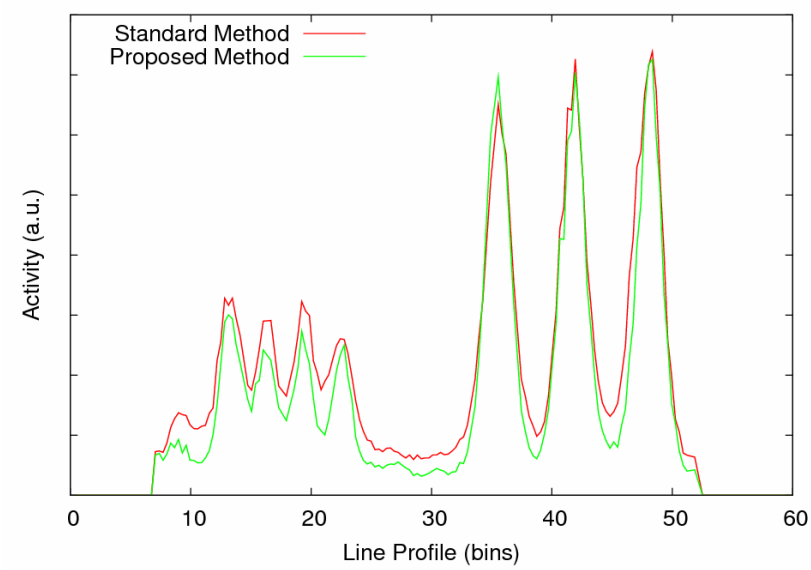

Fig. 6. Micro Deluxe Resolution phantom filled wiht FDG, acquired with rPET and reconstructed with the 3D-OSEM algorithm using standard (top left) and proposed (top right) methods. Improvements in resolution and peak to background ratios are clearly seen.
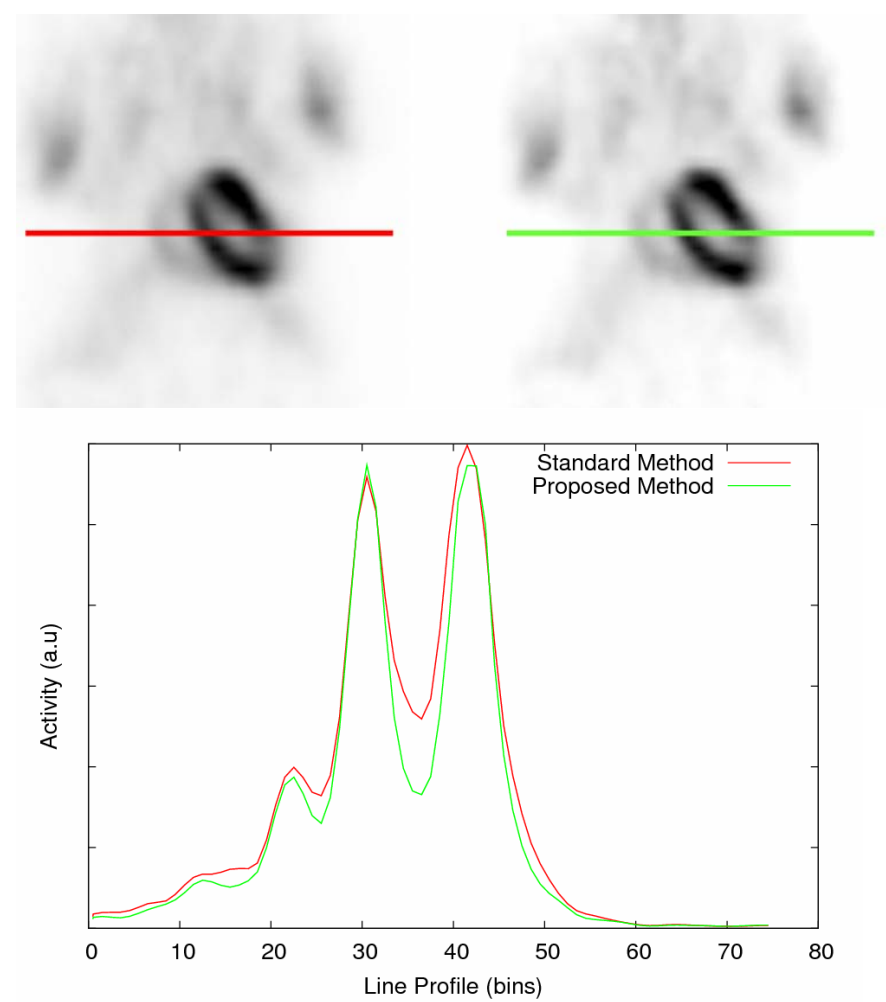

Fig. 7. Mouse injected with FDG and acquired with the rPET scanner. Both standard (top left) and improved (top right) method were employed and the 3D-OSEM reconstruction are compared. Line activity profiles plot (bottom) show the improvement achieved in the heart region.

\section{CONCLUSIONS}

The combination of XY position information with energy deposited in the detector, combined with a 3D-OSEM iterative reconstruction method, accelerates the convergence of the algorithm and allows to obtain better resolution and signal to background ratios in the image, without the need for additional hardware, at the expense of a moderate increase of noise level in uniform regions, due to the fact that counts with 
less reliable pixel identification are effectively removed from the reconstruction.

\section{ACKNOWLEDGMENT}

This work has been partially funded by projects TEC200407052-C02 of Ministerio de Educación y Ciencia and CDTEAM, program CENIT, Ministerio de Industria, Spain.

\section{REFERENCES}

[1] Seidel J., Vaquero J.J., Lee I.J., Green M.V,."Experimental Estimates of the Absolute Sensitivity of a Small Animal PET Scanner with Depth-ofInteraction Capability", IEEE Trans Nucl Sci, vol. 46, no. 3, pp. 485490, 1999.

[2] Lam C.F. et al, "Investigation of the effects of inter-crystal scatter reduction on reconstructed images in the jPET-D4 scanner", Vol. 24 No. 5, 318-327, 2005.

[3] S. Espana, J. L. Herraiz, J. J. Vaquero, M. Desco, and J. M. Udias, "PeneloPET: A MonteCarlo PET simulation tool based on PENELOPE," presented at IEEE Nuclear Science Symposium and Medical Imaging Conference, 2006.

[4] J. J. Vaquero, E. Lage, L. Ricon, M. Abella, E. Vicente, and M. Desco, "rPET Detectors Design and Data Processing," presented at IEEE Nuclear Science Symposium and Medical Imaging Conference, 2005.

[5] J. L. Herraiz, S. Espana, J. J. Vaquero, M. Desco, and J. M. Udias, "FIRST: Fast Iterative Reconstruction Software for (PET) tomography," Phys. Med. Biol., vol. 51, pp. 4547, 2006. Fan-Beam Collimators," Nuclear Science, IEEE Transactions on, vol. 50, pp. 398-404, $2003 .$. 